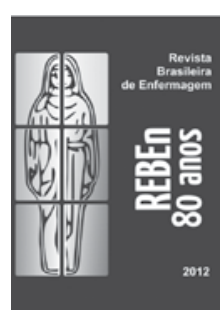

\title{
Panorama dos cursos de Graduação em Enfermagem no Brasil na década das Diretrizes Curriculares Nacionais
}

\author{
Overview of Nursing Graduation courses in Brazil in the National Curriculum Guidelines decade \\ Panorama de los cursos de Graduación en Enfermería en Brasil en el decenio de las Directrices \\ Curriculares Nacionales
}

\begin{abstract}
Elizabeth Teixeira', Josicelia Dumêt Fernandes", Andréia de Carvalho Andrade"'I', Kênia Lara Silvalv, Maria Eliane Martins Oliveira da Rocha ${ }^{\mathrm{V}}$, Raquel Josefina de Oliveira Lima ${ }^{\text {III }}$

' Universidade do Estado do Pará, Escola de Enfermagem Magalhães Barata. Belém-PA, Brasil.

"Universidade Federal da Bahia, Escola de Enfermagem, Departamento de Enfermagem Médico-Cirúrgica e Administração em Enfermagem. Salvador-BA, Brasil.

II' Universidade Federal de São Paulo, Escola de Enfermagem, Programa de Pós Graduação (Doutoranda). São Paulo-SP, Brasil.

IV Universidade Federal de Minas Gerais, Escola de Enfermagem. Belo Horizonte-MG, Brasil.

${ }^{v}$ Universidade Estadual do Piauí, Faculdade de Ciências Médicas, Curso de Enfermagem. Teresina-PI, Brasil.
\end{abstract}

Submissão: 29-07-2013 Aprovação: 29-07-2013

\begin{abstract}
RESUMO
O estudo teve o objetivo de construir um panorama dos cursos de Graduação em Enfermagem no Brasil, na década das Diretrizes Curriculares Nacionais do Curso de Graduação em Enfermagem, período 2001 a 2011. Trata-se de pesquisa exploratória, descritiva, com base em dados do e-MEC, Censo da Educação Superior, ENADE. Os resultados evidenciaram: privatização do ensino de Graduação em Enfermagem; excesso de oferta de cursos e vagas diurnas e noturnas; acentuada expansão do número de vagas de ensino à distância, não atendendo a critérios mínimos de qualidade avaliados pelo ENADE; dentre outros aspectos. Recomenda-se a definição de estratégias para regulação da expansão já instalada, além da revisão dos indicadores do Formulário Único do INEP, atendendo especificidades da Enfermagem, particularmente quanto a número de vagas, integração com o sistema local e regional de saúde e o SUS, ensino na área de saúde, atividades práticas de ensino, regime de trabalho e experiência profissional do corpo docente do curso. Recomenda-se, ainda, a intervenção imediata do MEC nos pólos de Educação à Distância, suspendendo a formação de enfermeiros nessa modalidade.

Descritores: Educação Superior; Educação em Enfermagem; Enfermagem.
\end{abstract}

\begin{abstract}
The study aims to build a general overview of the Nursing Graduation Programs in Brazil, in the decade of the National Curriculum Guidelines for Nursing Graduation, period 2001-2011. This is an exploratory, descriptive study, based on data from the e-MEC, Higher Education Census, ENADE. The results evidenced: the privatization of the Nursing Graduation Programs, the oversupply of courses and places day and night; sharp expansion of the number of vacancies for distance learning, not meeting the minimum quality criteria evaluated by ENADE, among other respects. It is recommended that strategies for the regulation of the expansion already installed must be defined, in addition to the revision of indicators drawn from the INEP Single Form, to meet the necessities of nursing, as an specific area, particularly with regard to: the number of vacancies, integration with the local and regional health system and the SUS, education in health care, teaching practical activities, working arrangements and experience of the faculty of the course. It is recommended, though, the immediate intervention of the MEC in the poles of Distance Education, suspending the training of nurses in that modality of education.
\end{abstract}

Key words: Higher Education; Nursing Education; Nursing. 


\section{RESUMEN}

El estudio objetivó construir una visión general de los Programas de Graduación en Enfermería en Brasil, en la década de los Lineamientos Curriculares Nacionales para Graduación en Enfermería, periodo 2001-2011. Estudio exploratorio, descriptivo, basado en datos del e-MEC, Censo de Educación Superior, ENADE. Los resultados evidenciaran: privatización de la Graduación en Enfermería; exceso de oferta de cursos y vagas nocturnas y diurnas; fuerte expansión del número de vagas de enseñanza a distancia, no cumpliendo criterios mínimos de calidad evaluados por ENADE, entre otros aspectos. Se recomienda la definición de estrategias para regulación de la expansión ya instalada, además de revisión de los indicadores del Formulario Individual INEP, para satisfacer la área de Enfermería, especialmente en relación con: número de vagas; integración con el sistema local y regionales de salud y la Seguridad Social; educación en el cuidado de la salud; enseñanza de actividades prácticas, régimen de trabajo y experiencia de los profesores del curso. Se recomienda, sin embargo, la intervención inmediata del MEC en los polos de educación a distancia, con suspensión de la formación de enfermeras en esa modalidad de educación.

Palabras clave: Educación Superior; Educación en Enfermería; Enfermería.

\section{AUTOR CORRESPONDENTE}

\section{INTRODUÇÃO}

Em atendimento à Carta de Belém, aprovada no $13^{\circ}$ Seminário Nacional de Diretrizes para a Educação em Enfermagem (SENADEn) e às Diretrizes do Movimento pela Qualidade da Formação dos Profissionais de Enfermagem (ABEn/COFEn), foi instituído um Grupo de Trabalho (GT), pela Associação Brasileira de Enfermagem (ABEn Nacional), com a finalidade de elaborar um panorama da Educação em Enfermagem no Brasil no período da década das Diretrizes Curriculares Nacionais (2001-2011) e, também, de definir estratégias e proposições que possibilitem a melhoria na qualidade da formação dos profissionais de Enfermagem, considerando a diversidade regional e a especificidade da área.

A constatação do acelerado e desordenado crescimento de cursos e oferta de vagas de graduação em Enfermagem sem o devido acompanhamento da sua qualidade indica a necessidade de estudos adicionais sobre a Educação em Enfermagem no Brasil.

A expansão da educação superior vem se dando, fundamentalmente, através do crescimento quantitativo do número de instituições de ensino e, conseqüentemente, de cursos e vagas por elas oferecidos. Essa expansão apresenta relação direta com o desenvolvimento econômico e social do país, uma vez que o Brasil apresenta baixa proporção de alunos, na faixa etária relevante, matriculados no ensino superior, não só em relação aos países mais desenvolvidos, mas também em relação aos países vizinhos da América Latina ${ }^{(1)}$.

Tais entendimentos expressam-se no processo que resultou na aprovação da Lei de Diretrizes e Bases da Educação Nacional (LDB) ${ }^{(2)}$ e que viabilizou a ampliação quantitativa de instituições de ensino superior no país, particularmente pela estratégia da interiorização (criação de instituições fora dos grandes centros) e de aumento de vagas nas grandes metrópoles.

Após a LDB, o Plano Nacional de Educação (PNE) ${ }^{(3)}$ apresentou uma política de Estado, para o decênio 2001-2010, estabelecendo, entre outras metas, uma ampliação da oferta de vagas em cursos superiores, compatível com a cobertura de $30 \%$ dos jovens brasileiros entre 18 e 24 anos.
O sistema educativo no país passou a apresentar grande crescimento da sua oferta, com a abertura de novos cursos, em sua maioria em instituições privadas e, muitas vezes, não atendendo as necessidades e demandas específicas de cada região, o que resultou na privatização do sistema e desigualdade geográfica ${ }^{(4-5)}$.

A política expansionista da educação superior, implementada no Brasil nas últimas décadas, reflete, pois, as exigências de mercado e pressões de grupos da sociedade civil para abertura de novos cursos e, neste cenário, destaca-se a possibilidade de formação superior no período noturno e na modalidade à distância.

A área da formação do enfermeiro acompanha essa tendência que, por sua vez, não parece garantir a formação de sujeitos críticos e reflexivos no atendimento à saúde integral do ser humano, conforme explicitado nas Diretrizes Curriculares Nacionais do Curso de Graduação em Enfermagem ${ }^{(6-7)}$.

Por outro lado, é importante pontuar que a necessidade de novos profissionais enfermeiros é uma realidade, considerando que a expansão de cursos e da oferta de vagas ainda é insuficiente para atender as múltiplas e diversas demandas de atenção à saúde da população nos níveis locais, regionais e nacionais ${ }^{(7)}$. Entretanto, ao tempo em que é necessário ampliar o número de enfermeiros no país, é necessário, também, não se descuidar dos parâmetros de qualidade acadêmica, ou seja, que esses profissionais sejam formados em cursos de qualidade reconhecida, que possam formar enfermeiros com perfil e competência para o atendimento às reais necessidades de saúde da população.

O GT ao problematizar essa realidade, levantou as seguintes questões: Qual o panorama da formação do enfermeiro, considerando a oferta dos cursos/vagas de graduação e a diversidade regional? O que aponta a avaliação do desempenho dos estudantes (ENADE 2010), segundo as notas obtidas pelos cursos? Quais os limites do processo de avaliação de cursos de graduação, considerando o formulário único do INEP?

Considerando a problemática acima delimitada, o estudo tem por objetivos: a) construir um panorama da formação e avaliação de Cursos de Graduação em Enfermagem no Brasil, período 2001 a 2011; b) definir estratégias para a regulação da expansão instalada de cursos de enfermagem. 


\section{METODOLOGIA}

Trata-se de pesquisa descritivo-exploratória ${ }^{(8)}$, cuja coleta de dados ocorreu no período de fevereiro a abril de 2013, por meio do site do e-MEC, Instituto Nacional de Pesquisa Anísio Teixeira (INEP), Conselho Federal de Enfermagem (Cofen), abrangendo os relatórios disponíveis do período de 2001 a 2011, período da primeira década das Diretrizes Curriculares Nacionais dos Cursos de Graduação em Enfermagem ${ }^{(6)}$.

Os dados foram organizados em um banco de dados criado com os recursos do programa Microsoft Excel@ para o cálculo da distribuição da frequência simples e relativa das variáveis de estudo: número de cursos e vagas presenciais, noturnos e à distância, número de ingressantes e concluintes nos cursos de Graduação em Enfermagem, proporção ingressantes e concluintes de enfermagem por habitante nas diferentes regiões; conceitos obtidos pelos cursos de graduação em enfermagem na avaliação do INEP/MEC.

Para a análise dos cursos noturnos, além da verificação na base de dados do INEP, foi realizada uma busca nas homepages das IES que ofertam esses cursos, investigando as atividades realizadas no período noturno (se teóricas ou práticas clínicas). Para a análise dos limites do processo de avaliação de cursos de Graduação em Enfermagem, foi feita uma consulta ao Formulário Único do INEP.

Os achados foram organizados em duas dimensões: Oferta dos cursos de graduação em enfermagem presenciais e à distância; e Avaliação dos cursos de Graduação em Enfermagem. Foi realizada, também, a análise no instrumento único de avaliação de cursos do INEP, considerando-se a especificidade da área de enfermagem, para, a partir daí, se propor a inserção de conteúdos específicos em sete dos indicadores que compõem $\mathrm{o}$ instrumento.

\section{RESULTADOS}

Oferta dos cursos de graduação em enfermagem presenciais e à distância

A análise dos dados do Censo da Educação Superior revelou um crescimento, dos anos 2001 a 2011, no número de cursos, número de vagas, número de ingressantes, inscritos e concluintes nos cursos de graduação em enfermagem no Brasil.

Em relação ao número de cursos, foi observado um incremento de $393 \%$ do número de cursos nas instituições privadas em comparação ao incremento de $122 \%$ em relação ao número de cursos públicos, no período analisado. Nesta comparação, o número de cursos de graduação em enfermagem no Brasil, em 2011, foi de 826, dos quais 160 em IES públicas e 666 em IES privadas. Em 2012 esse número foi de 838 cursos e, em 2013, o número de cursos atingiu 888 . No Gráfico 01, pode-se visualizar a distribuição dos cursos nas regiões do país.

O Gráfico 1 revela que o eixo Sul-Sudeste representa $71,2 \%$ do universo de cursos de Graduação em Enfermagem do Brasil. Nestas regiões, destacam-se os estados de São Paulo, Rio de Janeiro, Minas Gerais e Paraná, com o maior número de cursos. As regiões Norte e Centro-Oeste apresentaram 17\% dos cursos de graduação em Enfermagem do país. Em toda a década, o número de cursos privados foi superior ao número de cursos públicos, chegando a uma relação de 4,16 cursos em IES privadas para cada curso em IES pública. O Gráfico 2 retrata esse achado, demonstrando que a concentração dos cursos em IES privadas localiza-se nas regiões Sudeste e Sul. Nas demais regiões, estes cursos estão presentes em maior proporção nos Estados com maior contingente população e desenvolvimento econômico tais como Bahia, no Nordeste e Goiás no Centro Oeste.

Gráfico 1 - Oferta de Cursos de graduação em Enfermagem por Região Geográfica 1991-2012

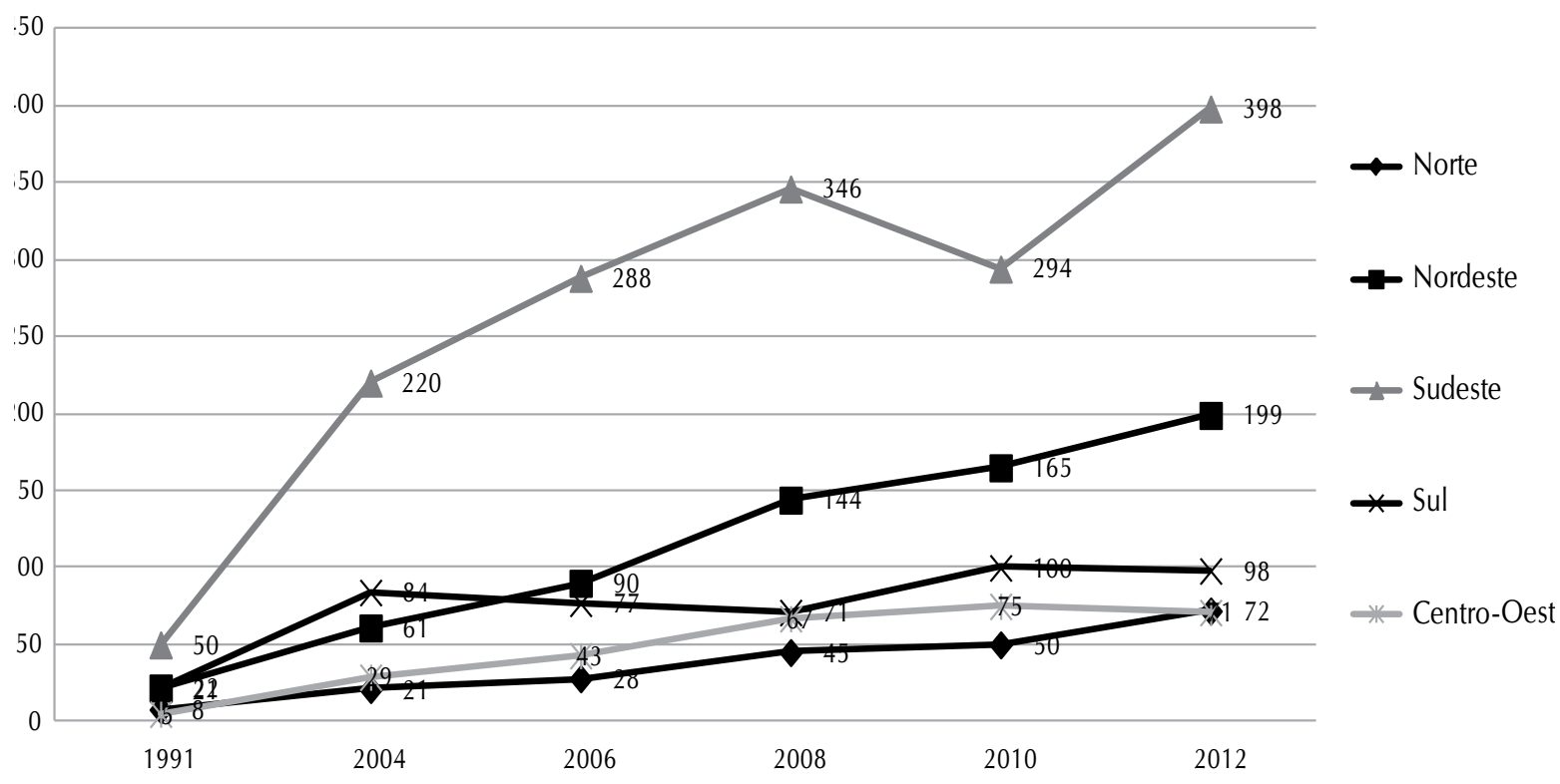


Gráfico 2 - Oferta de cursos de graduação em enfermagem por dependência administrativa. Brasil - 2011.

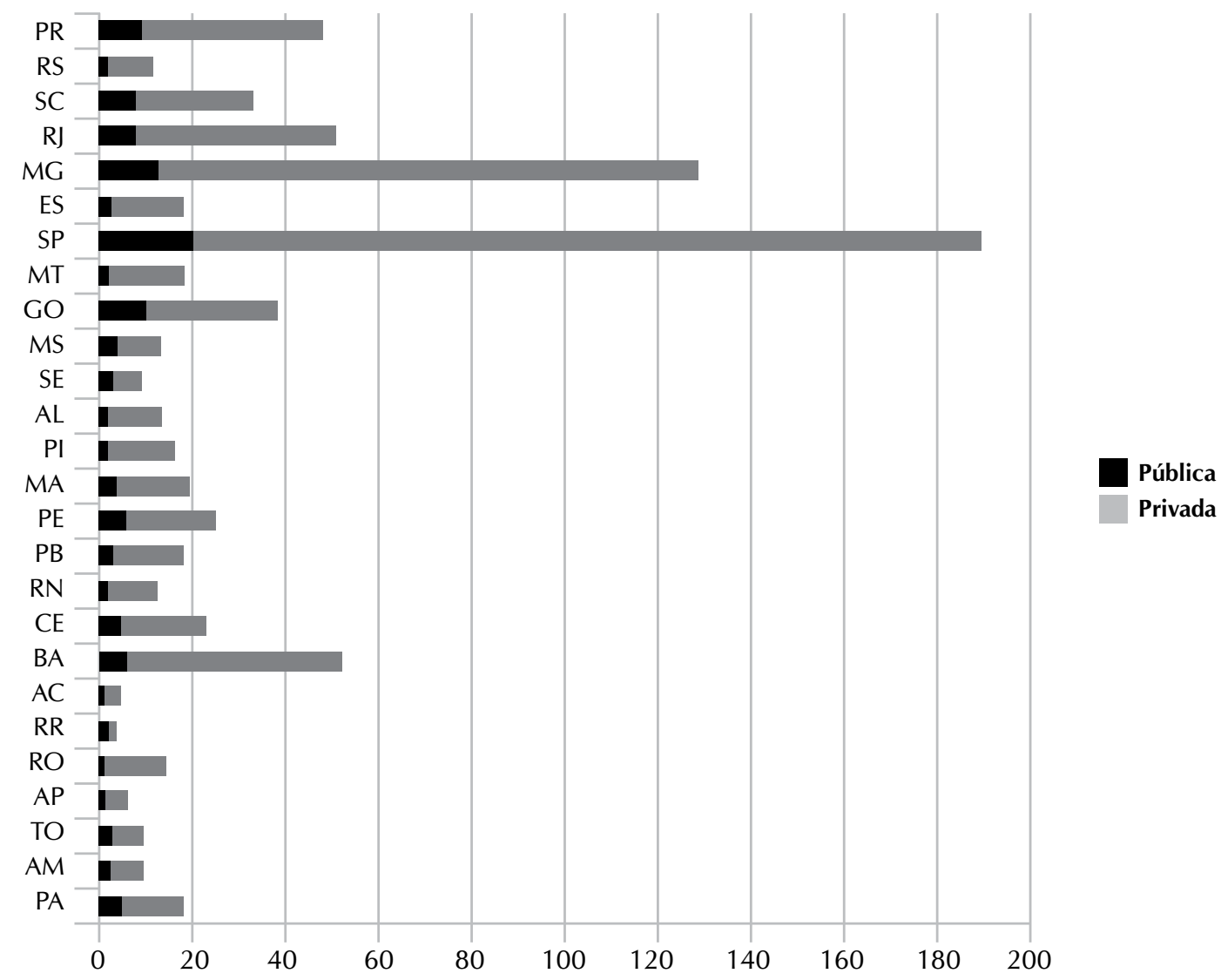

\begin{tabular}{|c|c|c|c|c|c|c|c|c|c|c|c|c|c|c|c|c|c|c|c|c|c|c|c|c|c|c|}
\hline & PA & AM & TO & AP & RO & RR & AC & BA & CE & RN & PB & PE & MA & PI & AL & SE & MS & GO & MT & SP & ES & MG & RJ & SC & RS & PR \\
\hline Pública & 5 & 2 & 3 & 1 & 1 & 2 & 1 & 6 & 5 & 2 & 3 & 6 & 4 & 2 & 2 & 3 & 4 & 10 & 2 & 20 & 3 & 13 & 8 & 8 & 2 & 9 \\
\hline \begin{tabular}{|l|} 
Privada \\
\end{tabular} & 13 & 7 & 6 & 5 & 13 & 1 & 3 & 46 & 18 & 10 & 15 & 19 & 15 & 14 & 11 & 6 & 9 & 28 & 16 & 170 & 15 & 116 & 43 & 25 & 9 & 39 \\
\hline
\end{tabular}

Tabela 1 - Número de cursos, vagas, inscritos, ingressantes, concluintes e matriculados nos cursos de Graduação em Enfermagem no Brasil, 2001-2011.

\begin{tabular}{|c|c|c|c|c|c|c|c|c|c|c|c|}
\hline & $\mathbf{2 0 0 1}$ & $\mathbf{2 0 0 2}$ & $\mathbf{2 0 0 3}$ & $\mathbf{2 0 0 4}$ & $\mathbf{2 0 0 5}$ & $\mathbf{2 0 0 6}$ & $\mathbf{2 0 0 7}$ & $\mathbf{2 0 0 8}$ & $\mathbf{2 0 0 9}$ & $\mathbf{2 0 1 0}$ & $\mathbf{2 0 1 1}$ \\
\hline Cursos públicos & 72 & 81 & 86 & 91 & 96 & 114 & 124 & 132 & 148 & 161 & 160 \\
\hline Cursos privados & 135 & 194 & 239 & 320 & 373 & 443 & 505 & 547 & 604 & 638 & 666 \\
\hline TOTAL CURSOS & 207 & 275 & 325 & 411 & 469 & 557 & 629 & 679 & 752 & 799 & 826 \\
\hline TOTAL VAGAS & 23946 & 36221 & 46687 & 70240 & 83159 & 94818 & 107691 & 115187 & 118616 & 116343 & 119753 \\
\hline TOTAL INSCRITOS & 119938 & 183753 & 198376 & 229174 & 221620 & 239859 & 236128 & 230466 & 255865 & 257374 & 295648 \\
\hline TOTAL INGRESSOS & 20942 & 30275 & 38202 & 50668 & 58174 & 61775 & 66836 & 65006 & 65001 & 60423 & 70175 \\
\hline TOTAL CONCLUINTES & 6177 & 8901 & 11069 & 13816 & 19813 & 25582 & 32280 & 38973 & 40692 & 42853 & 47114 \\
\hline TOTAL MATRICULAS & 51905 & 69955 & 90739 & 119920 & 152276 & 185902 & 211523 & 222615 & 234070 & 244568 & 244245 \\
\hline
\end{tabular}


Em relação ao número de vagas, foi observado que, em 2011, existiam 119.753 vagas, o que representou um incremento de $400 \%$ em relação àquelas existentes em 2001, que perfaziam 23.946 vagas. O crescimento médio foi de 9.580 vagas/ano, com oscilações negativas entre os anos de 2009/2010.

Os dados revelaram a variação entre vagas, inscritos, ingressos e concluintes nos cursos de graduação em enfermagem no Brasil na década 2001-2011. Nesta relação, a proporção inscritos/vagas mostrou-se positiva durante toda a década, com 5 inscritos para cada vaga em 2001 decrescendo para 2,46 inscritos para cada vaga em 2011. Essa queda começou a ser observada a partir do ano de 2003 , indicando uma diminuição na procura pelos cursos de enfermagem.

A relação vagas/ingressantes também demonstrou valores positivos durante toda a década, variando de 1,14 em 2001 a 1,7 em 2011, com maior índice em 2010 quando se observou 1,92 vagas para cada ingressante. A maior taxa de aproveitamento das vagas ocorreu no início da década. As vagas ociosas nos cursos de graduação em enfermagem representaram $37 \%$ das vagas ofertadas, com uma tendência de crescimento ao longo de toda a década, indicando que a abertura de novas vagas se deu sem controle e análise da oferta e demanda.

A ampliação do número de concluintes nos cursos de graduação em enfermagem é consequência do aumento dos ingressantes. Ainda que não se possa estabelecer uma relação direta, temporal, entre aqueles que ingressaram nos cursos em determinado ano e a sua conclusão, o que se observou, durante toda a década, foi a evasão nos cursos de graduação expressa pelo número de concluintes sempre menor que o de ingressantes.
Em 2001, a proporção de concluintes/ingressos foi de 30\%, melhorando essa relação em 2008, quando se passou a observar $60 \%$ na proporção entre concluintes e ingressantes. Vale ressaltar que o número de concluintes no setor público se manteve quase que inalterado devido à estagnação do número de cursos e vagas nesse setor, durante a década estudada. A expansão da Graduação em Enfermagem vem ocorrendo não apenas nos cursos diurnos, mas também nos cursos noturnos.

Os cursos presenciais disponibilizaram, em 2011, 54.718 vagas no período noturno, o que correspondeu a $45,69 \%$ do total de vagas presenciais dos cursos de graduação em enfermagem. A maior concentração de cursos e vagas foi na região sudeste e, mais especificamente, nos estados de São Paulo, Minas Gerais e Rio de Janeiro, responsáveis por cerca de, $59 \%$ do total das vagas no período noturno.

Chamou a atenção o fato de que, no Brasil, são oferecidas 19. 680 vagas em Educação à Distância, por meio de 291 pólos com oferta de cursos de Graduação em Enfermagem, por duas instituições - A e B. A instituição A oferece 16.800 vagas distribuídas em 240 pólos e a $B$ oferece 2.880 vagas distribuídas em 51 pólos.

O Gráfico 04 demonstrou que o maior número de pólos de EAD para cursos de graduação em enfermagem encontra-se na região sudeste, onde a instituição $A$ conta com 85 pólos (72\%) e a instituição B com 37 pólos (35\%). O menor número de pólos está presente na região norte. Foi observada uma predominância da instituição A na maior parte do território nacional, exceto no Rio de Janeiro (32), onde houve maior predomínio da instituição B.

Gráfico 03 - Distribuição geográfica do número de cursos e vagas no período noturno segundo unidades da federação. São Paulo, 2013.

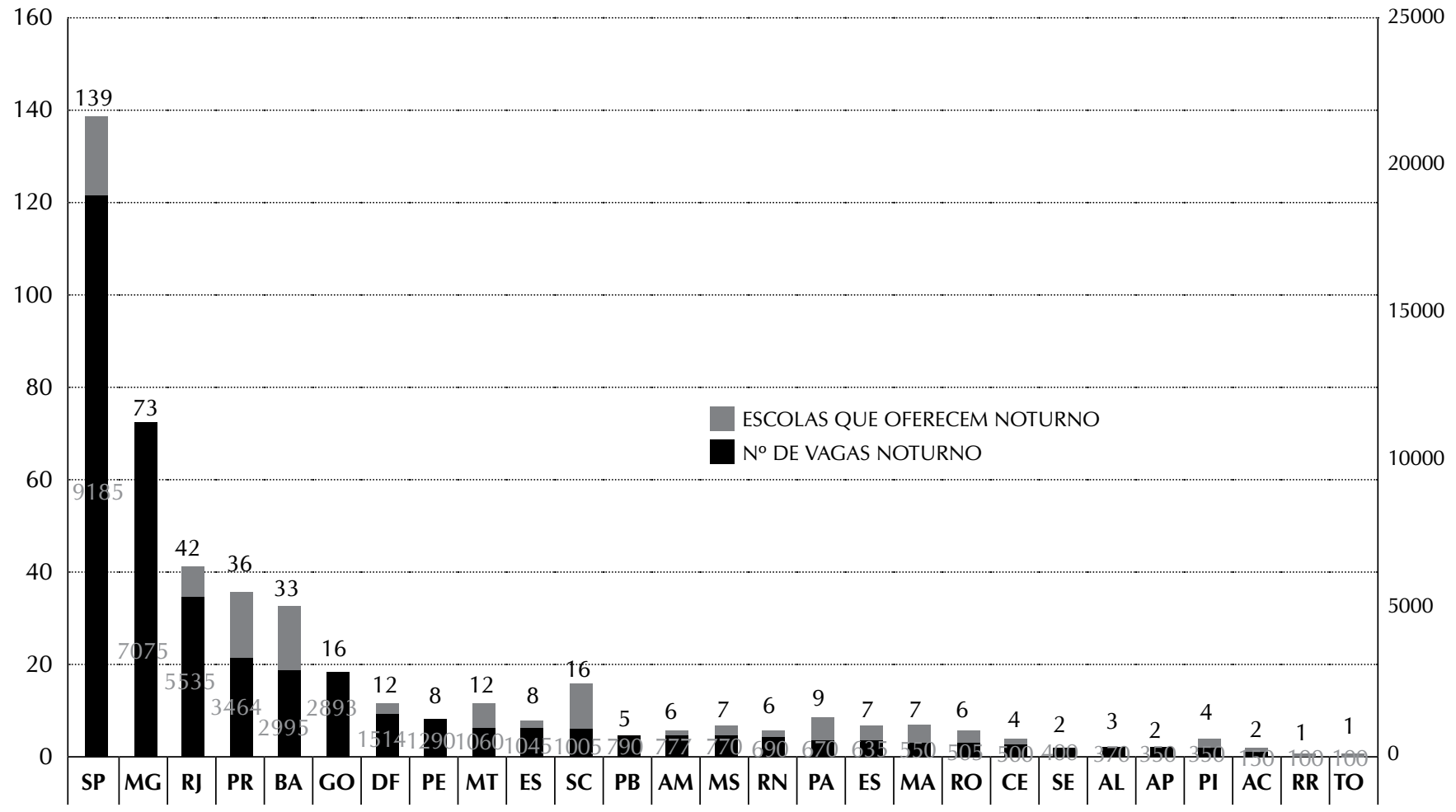


Gráfico 4 - Distribuição dos polos de Educação à Distância, por universidade e unidades da federação. Brasil, 2013.

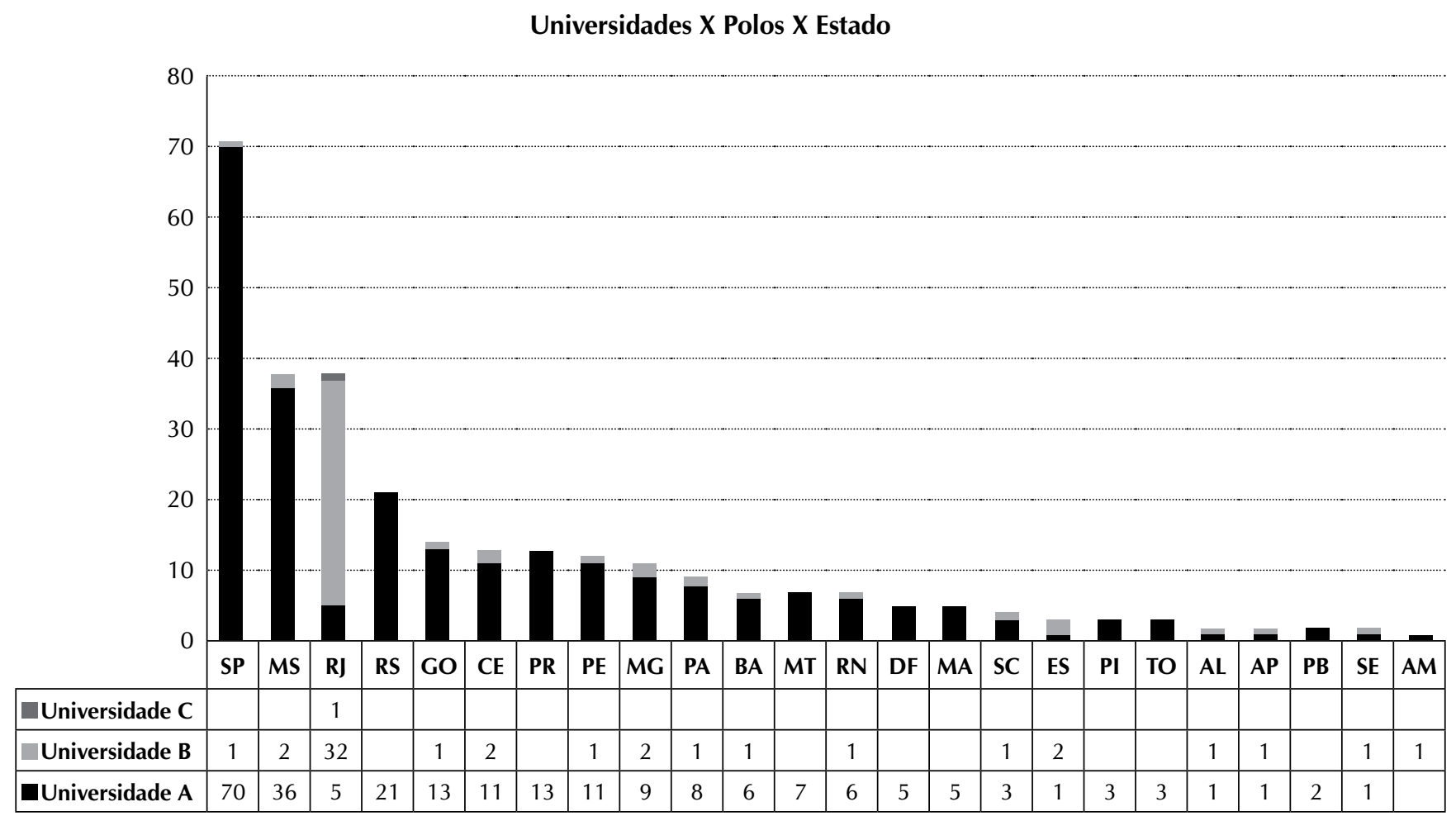

Avaliação dos cursos de enfermagem

Sobre o ENADE 2010, ao se analisar o total de cursos de Enfermagem presenciais e existentes até março de 2013, foi observado que do total de 888 cursos registrados em março 2013, no e-MEC, 546 tinham notas consolidadas na avaliação ENADE 2010, 61,5\% do total; 342 cursos estavam ainda sem informação ou sem conceito, sendo $167(18,8 \%)$ sem conceito (SC) e $175(19,7 \%)$ não tinham ainda parâmetros para avaliação. No resultado global de notas ENADE 2010, foi verificado que as notas 1 e 2 obtidas pelos alunos na prova do ENADE totalizaram $43 \%$, dos cursos; seguido pela nota 3, com $36 \%$. As notas 4 e 5 equivaleram a $21 \%$.

Gráfico 05 - Distribuição das notas do ENADE 2010 nos cursos de Enfermagem por região do país. Brasil, 2013.

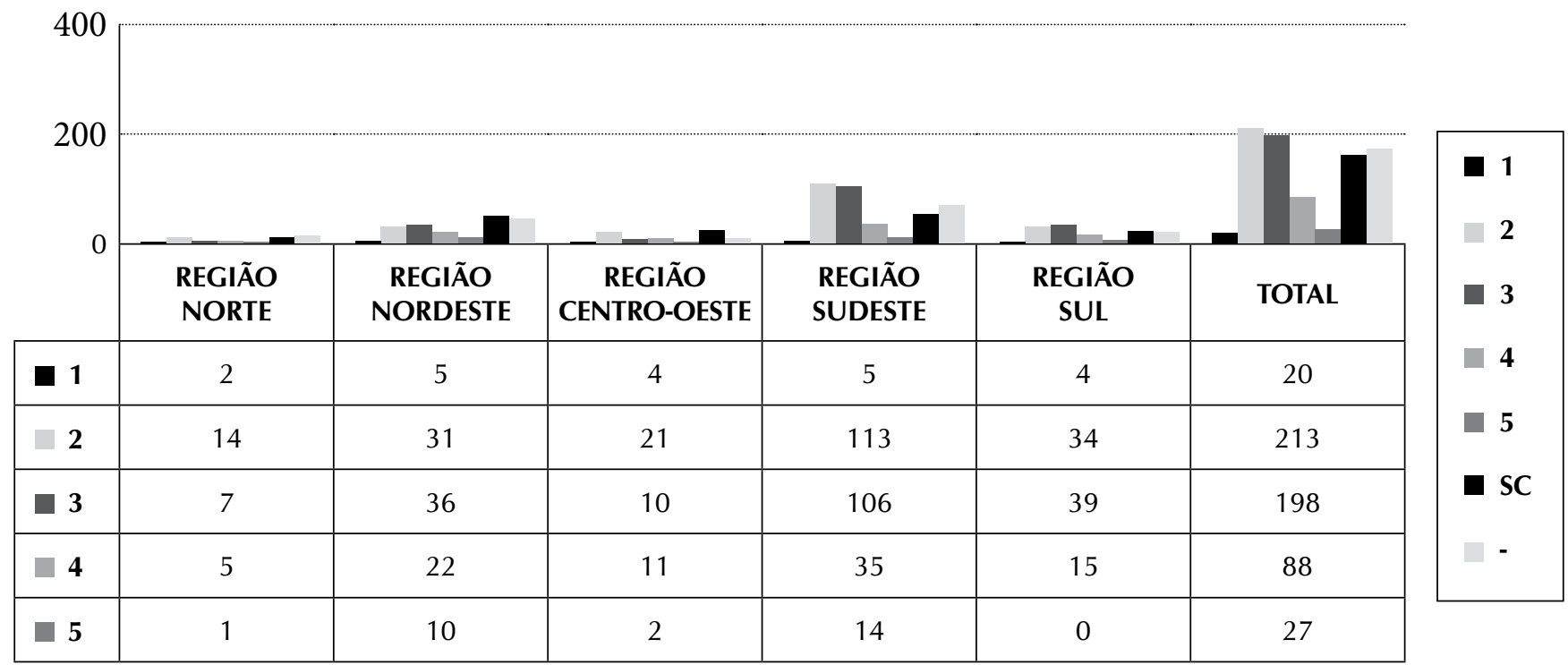


O Formulário Único do INEP, utilizado para os atos de Autorização, Reconhecimento e Renovação de Reconhecimento, está organizado em 3 Dimensões. Em cada uma das Dimensões foi constatado que há indicadores que só são obrigatórios para os Cursos de Medicina, em que os avaliadores de Enfermagem registram Não Se Aplica (NSA). Essa constatação indicou a necessidade de construção de indicadores específicos, também, para a área de enfermagem.

\section{DISCUSSÃO}

No contexto brasileiro, a década de 2001-2011 representou um importante momento de reestruturação da formação de enfermeiros a partir da implantação das Diretrizes Nacionais Curriculares (DCN) em 2001. As DCN representam um instrumento norteador do processo de construção dos Projetos Político-Pedagógicos (PPP) e revelam as bases filosóficas, conceituais, políticas e metodológicas do processo de formação. A implantação dessas Diretrizes tem sido assumida como um marco estruturante na construção de um novo paradigma para a educação de enfermagem: a orientação da formação para impulsionar a efetivação dos princípios do SUS e das demandas e necessidades de saúde da população ${ }^{(9-10)}$.

Neste contexto, os achados corroboram outros estudos que demonstram trajetórias de expansão na formação de enfermeiros e outros profissionais de saúde no país ${ }^{(11-14)}$. A expansão é reflexo do aumento no número de cursos/vagas em instituições públicas e privadas e da ampliação do acesso populacional à educação superior no país ${ }^{(15-16)}$.

A privatização do ensino de graduação em enfermagem é uma marca da década, confirmando outros estudos que apontaram essa tendência nos anos recentes para o ensino de enfermagem no Brasil ${ }^{(11,17)}$

A privatização é reflexo da conformação do sistema educativo no país que passou a apresentar grande crescimento da sua oferta, com a abertura indiscriminada de novos cursos, sem controle, por exemplo, da necessidade e demandas específicas de cada região, ocasionando uma desigualdade geográfica (desequilíbrio regional e intra-regional) dessa oferta e, ainda, a privatização do sistema, além da proliferação descontrolada de cursos e instituições cada vez mais distantes da ideia de universidade e de padrões mínimos de qualidade ${ }^{(4)}$.

Outros estudos sobre a formação de profissionais e a distribuição de recursos humanos de saúde também revelam essa desigualdade identificando que nas regiões Sul e Sudeste do país há melhores índices na relação egresso/habitante dos cursos de graduação da área de saúde enquanto as regiões Norte e Nordeste possuem os piores índices ${ }^{(12,18)}$.

Mesmo superado o desafio da distribuição regional de cursos e vagas, há ainda outros elementos que merecem discussão no complexo fenômeno do panorama da educação em enfermagem no Brasil. O primeiro deles refere-se ao "desperdício de vagas" observado pela desproporção entre vagas ofertadas $x$ ingressantes $x$ concluintes nos cursos de graduação em enfermagem.

Neste sentido, há indicativos que, sobretudo, as vagas ociosas estão nas instituições de ensino de caráter privado. $\mathrm{O}$ que se revela é um ciclo de desvantagens para aqueles alunos trabalhadores que acabam realizando uma dupla jornada - a de profissional e a de graduando de enfermagem - com importantes limitações para dedicar-se à sua formação. Uma das consequências é a evasão do curso, o que representa para o sistema público de ensino, um desperdício de recursos e, por outro lado, para o sistema privado uma economia de recursos, uma vez que o investimento inicial do aluno não é devolvido quando este "abandona" a formação.

O segundo aspecto refere-se à expansão dos cursos noturnos e à necessidade de se discutir a adequação e qualidade desta formação. Ainda que os cursos sejam registrados e ofertados, predominantemente, no período noturno, o que se observa é o desenvolvimento do ensino clínico, práticas e estágios no período diurno. Contudo, nem sempre essas informações estão disponíveis para aqueles que procuram pelos cursos. Esse pode ser um dos fatores que determina a evasão nos cursos, especialmente dos alunos trabalhadores, reproduzindo, com isso, o ciclo de desvantagens.

Há que se discutir, também, a oferta de cursos de Graduação em Enfermagem na modalidade à distância. Entende-se que a educação à distância pode ser uma importante estratégia de democratização do ensino, em especial naquelas regiões de difícil acesso à educação no país. Contraditoriamente, o ensino à distância em saúde e enfermagem, ainda que autorizado por Portaria Ministerial(19), aponta para o desafio de utilizar esta modalidade, em que professor e aluno não se encontram presentes fisicamente, na mesma hora e no mesmo espaço geográfico, para desenvolver a característica intrínseca da formação da Enfermagem que requer, para o desenvolvimento das competências e habilidades profissionais, o contato humano e presencial, em trocas que se estabelecem no ato de cuidar.

Associa-se a essa discussão, o reforço das desigualdades que a oferta dos cursos de graduação de enfermagem à distância provoca ao se constatar que a região com maior número de pólos, também é a região com maior número de vagas para o curso presencial e aquela com melhores índices de desenvolvimento socioeconômico.

Por fim, a avaliação dos cursos de é um aspecto complexo do fenômeno panorama da educação em enfermagem. Cabe destacar que a qualidade de um curso de graduação em enfermagem envolve múltiplas dimensões que se interpenetram e requerem uma visão de totalidade para lograr uma aproximação com a realidade concreta onde esses cursos se inserem. Nessa aproximação, o olhar dos sujeitos do processo de formação merece ser ressaltado, como parte de um diagnóstico que nos desafia a problematizar os dados obtidos como forma de subsidiar o processo de aprimoramento da qualidade do ensino na condição de docentes e/ou gestores dos cursos.

O Exame Nacional de Desempenho dos Estudantes (ENADE), uma das modalidades de avaliação do Sistema Nacional de Avaliação da Educação Superior (SINAES) realizado no Brasil, tem como objetivos: aferir o desempenho dos estudantes em relação aos conteúdos programáticos previstos nas Diretrizes Curriculares, suas habilidades para ajustamento às exigências decorrentes da evolução do conhecimento e suas 
competências para compreender temas exteriores ao âmbito específico de sua profissão.

Estudantes, ingressantes e concluintes de cursos de todo o país, em 2004, 2007 e 2010, realizaram o exame. Os estudantes são sorteados aleatoriamente pelo Instituto Nacional de Estudos e Pesquisas Educacionais Pedagógicos (INEP), do Ministério da Educação (MEC), e além de realizarem uma prova de conteúdos gerais e específicos, respondem um questionário-pesquisa com questões sobre seu perfil e indicadores de qualidade de seu Curso de Graduação em Enfermagem.

Verifica-se, no entanto, que, no relatório do ENADE 2010, a maioria dos cursos não atendiam aos critérios mínimos de qualidade. Assim, há que se rever sete indicadores do Formulário Único do INEP, para atender especificidades da Enfermagem, a saber: DIMENSÃO 1 - 1.18. Número de vagas; 1.20. Integração com o sistema local e regional de saúde e o SUS; 1.21. Ensino na área de saúde; 1.22. Atividades práticas de ensino. DIMENSÃO 2 - 2.9. Regime de trabalho do corpo docente do curso; 2.10. Experiência profissional do corpo docente; 2.12. Experiência de magistério superior do corpo docente.

\section{CONSIDERAÇÕES FINAIS}

A construção do panorama da formação e avaliação de Cursos de Graduação em Enfermagem no Brasil, período
2001 a 2011 indicou uma expansão instalada que precisa ser regulada. Assim, emerge um conjunto de estratégias, com vistas à formulação de políticas de formação profissional de Enfermagem.

Uma das estratégias, apontada na CARTA de BELEM, e que o GT reforça, é a inclusão da Enfermagem entre as profissões cujos pedidos de autorização, reconhecimento e renovação de reconhecimento de Cursos de Graduação em Enfermagem são submetidos a prévia manifestação do Conselho Nacional de Saúde-CNS/CIRH, conforme já ocorre com os cursos de Medicina, Odontologia e Psicologia, previstos no art. 10 do Decreto $n^{\circ}$ 5.840, de 13 de julho de 2006, que modificou a redação do parágrafo $2^{\circ}$ do art. 28 do Decreto $n^{\circ} 5.773$, de 9 de maio de 2006.

Outra estratégia que o GT reforça é o urgente redimensionamento da oferta de vagas nos Cursos/Faculdades/Escolas de Enfermagem, tomando-se como marco de referência as necessidades sociais em saúde da população, do mercado de trabalho e da oferta de espaços de formação clínica para os estudantes de enfermagem, conforme previsto na Resolução 350 do Conselho Nacional de Saúde.

O GT também é a favor da intervenção imediata do MEC nos 291 polos de Educação à Distância, desenvolvidos por duas IES privadas no país, suspendendo a formação de enfermeiros nessa modalidade.

\section{REFERÊNCIAS}

1. Zarur GCL. O discurso liberal e a política de expansão da educação superior no Brasil [artigo na internet] [acesso em 29 jul 2013]. Disponível em: http://www.georgezarur. com.br/pagina.php/63 Acessado em 15/4/2011.

2. Brasil. Ministério da Educação. Lei n 9.394, de 20 de dezembro de 1996. Estabelece as diretrizes e bases da educação nacional. Diário Oficial da União 23 dez 1996;34(248)Seção 1:27.833-41.

3. Brasil. Ministério da Educação. Lei $n^{\circ} 10.172$, de 9 de janeiro de 2001. Aprova o Plano Nacional de Educação e dá outras providencias. Diário Oficial da União 10 jan 2001.

4. Oliveira JF, Dourado LF, Amara NC, Moehelecke S, Catani AM. Políticas de acesso e expansão da educação superior: concepções e desafios. Brasília (DF): MEC/INEP; 2006.

5. Fernandes JD. Diretrizes Curriculares e Projetos Pedagógicos de cursos de graduação em enfermagem. In: Teixeira E, Vale EG, Fernandes JD, Sordi MRL,organizadores. O ensino de graduação em enfermagem no Brasil: o ontem, o hoje e o amanhã. Brasília: INEP; 2006.

6. Ministério da Educação (Brasil). Resolução CNE/CES nº.3, de 7 de novembro de 2001. Diretrizes Curriculares Nacionais do Curso de Graduação em Enfermagem.Diário Oficial da União 2001; Seção 1.

7. Teixeira E, Vale EG. Tendências e perspectivas do ensino de graduação em enfermagem. In: Teixeira E,Vale EG, Fernandes JD, Sordi MRL, organizadores. O ensino de graduação em enfermagem no Brasil: o ontem, o hoje e o amanhã. Brasília: INEP; 2006

8. Gil AC. Métodos e técnicas de pesquisa social. São Paulo: Atlas; 2008

9. Silva KL, Sena RR. Integralidade do cuidado na saúde: indicações a partir da formação do enfermeiro. Rev Esc Enferm USP 2008;42(1):48-56.

10. Lopes Neto D. Aderência dos Cursos de Graduação em Enfermagem às Diretrizes Curriculares Nacionais. Rev Bras Enferm 2007;60(6):27-34.

11. Rodrigues RCV, Peres HHC. Panorama brasileiro do ensino de enfermagem on-line. Rev Esc Enferm USP 2008;42(2):299-304

12. Haddad AE, Morita MC, Pierantoni CR, Brenelli SL, Passarella T, Campos FE. Formação de profissionais de saúde no Brasil: uma análise no período de 1991 a 2008. Rev Saúde Pública 2010;44(3):383-393.

13. Vieira ALS, Garcia ACP, Filho AA, Pierantoni CR, Ferraz $\mathrm{CP}$, Oliveira ES, et al. Tendências do sistema educativo no Brasil: medicina, enfermagem e odontología. In: Ministério da Saúde. Observatório de Recursos Humanos em Saúde no Brasil: estudos e análises. Brasília: Ministério da Saúde; 2004.

14. Teixeira E, Vale EG, Fernandes JD, Sordi MRL. Trajetória e tendências dos cursos de enfermagem no Brasil. Rev Bras Enferm 2006;59(4):479-487

15. Silva KL, Sena RR, Tavares TS, Martins ACS. Oferta dos 
cursos de graduação em enfermagem no estado de Minas Gerais. Texto \& Contexto Enferm 2011;20(nº.esp):124-30.

16. Rodrigues RM, Caldeira $S$. Formação na graduação em enfermagem no estado do Paraná. Rev Bras Enferm 2009;62(3):417-23.

17. Silva KL, Sena RR, Tavares TS, Wan der Maas L. Expansão dos cursos de graduação em enfermagem e mercado de trabalho: reproduzindo desigualdades? Rev Bras
Enferm 2012; 65(3):406-13.

18. Ceccim RB, Pinto LF. A formação e especialização de profissionais de saúde e a necessidade política de enfrentar as desigualdades sociais e regionais. Rev Bras Educ Med 2007; 31(3):266-277

19. Brasil. Decreto $n^{\circ} .6 .303$, de 12 de dezembro de 2007. Estabelece as diretrizes e bases da educação nacional. Diário Oficial da União 13 dez 2007: Seção 1. p. 4. 